Innovations in Incidence Geometry

Volume 15 (2017), Pages 287-297

ISSN 1781-6475

\title{
Happy 70th, Jef
}

\section{Stanley E. Payne}

I thank the organizers of this conference for including me in this birthday celebration. My remarks will recall a few personal memories of times that I enjoyed with Jef and his family, and then end with a few purely mathematical remarks. Most of you here are already familiar with much of his work, so I feel that I can safely ignore the definitions of many of the terms that will appear in what follows. I want to make it clear that I am immensely grateful for my interaction with Jef and that I hold him in very high esteem.

In the late 1960's I began to study generalized quadrangles, totally unaware that anyone else in the world was working on them. So it was quite a surprise to me when in January of 1972 I received a letter from Prof. Jacques Tits asking me to referee a paper written by someone named Josef A.Thas. Professor Tits stressed quite strongly the importance of remaining anonymous. The paper seemed to me to be one that I should have written myself, and it was a very pleasant for me to discover someone else was interested in GQ. I waited some time to write to this Professor Thas, because I did not want him to think I had been the one to referee his paper. That was a bit ironic because a couple years later I discovered that his entire department was convinced that I had to have been the referee. When he informed me that he had refereed a paper of mine I was forced to admit my own involvement with his paper.

We began to correspond by snail mail with hand-written letters. Since he would address me as Prof. Dr. S. E. Payne I felt obliged to address him as Prof. Dr. J. A. Thas. For a mid-western American this formality seemed quite unnatural. So eventually I started a letter to him by saying: "Hi, Joe," because Joe seemed to me to be the natural diminutive for the name Josef. His next letter to me started: "Hi, Stan" and was signed by Joe.

My first trip to Europe was in 1974 and my first stop was in Brussels at the Free University. Bill Kantor, Ernie Shult and I each gave talks before I had any real opportunity to meet anyone from Ghent. In my talk I cited some work by someone named Joe and afterwards Francis Buekenhout asked me who was this Joe I had mentioned. I was quite surprised to discover that Professor Dr. J. A. Thas was well known as Jef, and I was possibly the only one in the world 
for whom he was Joe. (I have since learned that a number of Americans and Canadians refer to him as Joe.) After many trips to Europe and being with his other friends I have gradually become accustomed to calling him Jef, but in my private thoughts he will always be Joe.

During that first trip to Europe in 1974 Jef and Laurette made sure that I was treated better than I had ever been treated in my own country. So it was that in 1974 there began a fruitful collaboration with Jef that eventually led to the publication of the book Finite Generalized Quadrangles, popularly known as the red book [PT84]. That was a true labor of love. Working with Jef was just perfect. We each prepared drafts of different parts of the book, with each of us concentrating on those parts most closely associated with the other person. This attempt to avoid mistakes paid off! Jef found that there was a gap in my published proof of the uniqueness of the GQ of order 4. We spent perhaps three months unsuccessfully trying to fill in the gap before taking our problem to Jacques Tits. He solved the problem in three days and the resulting complete proof appeared in the red book. In 2009 a second edition appeared in a bright blue cover [PT09]. I wonder if it will still be called the red book?

To collaborate with Jef was (and still is) just fantastic! Every time I asked him a question or sent him an idea or an introduction to a topic about GQ, he responded with more ideas and better results, so that theorems just seemed to flow into a paper. On this occasion, as we celebrate Jef's 70th birthday, I want to mention some of the very many results that he has discovered and taught to the rest of us over the years. I hope you forgive me for restricting my attention to a couple results on generalized quadrangles closely related to my own work.

In the fall of 1987, just prior to a conference in Lincoln, Nebraska, that we were both about to attend, Jef visited me in my home in the mountains above Denver. I had recently shown that the so-called flock generalized quadrangles with parameters $\left(q^{2}, q\right)$ satisfy a property I called Property (G), although it had not yet been written up in a paper. In a general not very precise sense this meant that there was the maximum possible number of $(q+1) \times(q+1)$ grids covering a certain point denoted $(\infty)$. I showed Jef how to construct an affine space using these grids and conjectured that this might be the first step in proving that a generalized quadrangle satisfying Property (G) at a point must be a flock generalized quadrangle. Actually, I was constructing these GQ from sets of matrices that I called $q$-clans. It was Jef who discovered the connection with flocks of quadratic cones, and eventually showed how to construct them directly from the conical flocks, so that they became known as flock GQ. It took a decade and much hard work, but Jef eventually proved this conjecture using ideas from algebraic geometry. At first he needed a small additional hypothesis in characteristic 2 , but eventually managed to overcome even that little problem. I think 
this was a truly first rate accomplishment! However, my knowledge of algebraic geometry was (and is) nearly nonexistent, so I was quite relieved when some Australians came up with a proof that was a more elementary combinatorial and geometric proof. Matt Brown was the one who first completed the proof in a final form that I could fully understand, but Jef was there first and really inspired the others.

The second item I want to mention is really an entire joint paper with Jef that contains several results that continue to give me pleasure. The paper is: "Spreads and ovoids in finite generalized quadrangles," Geom. Dedicata $\mathbf{5 2}$ (1994), 227-253 [PT94]. I particularly want to mention a construction of a new family of ovoids in the classical generalized quadrangles $Q\left(4,3^{e}\right), e \geq 3$. In 1983 as I was trying to complete the manuscript of what would become the red book, Jef sent me a new theorem for inclusion. Starting with a translation generalized quadrangle Jef constructed another one now called its translation dual (see Theorem 8.7.2 part (iv) of the red book). At that time we had not begun seriously to investigate whether or not the known translation GQ would give new translation duals. However, I spent the month of January 1988 in Rome as a guest of Marialuisa, living in a flat that belonged to James Hirschfeld. At the urging of Marialuisa I completed a proof that a certain translation GQ $\mathcal{S}$ with parameters $\left(q, q^{2}\right)$ with $q=3^{e}(e \geq 3)$ had a translation dual $\mathcal{S}^{D}$ that was new and different in several ways from all examples known before. The starting point GQ was the point-line dual of a flock GQ that arose by a connection discovered by Jef and Norm Johnson from a family of translation projective planes originally constructed by M. Ganley. I named the newly constructed translation GQ the Roman GQ. They played a role in our joint paper on spreads and ovoids.

If $\mathcal{S}$ is a translation GQ with parameters $(s, t)$ where $s \neq t$, then we know there is a prime $p$ such that $q$ is a power of $p$ and $(s, t)=\left(q^{a}, q^{a+1}\right)$ for some odd integer $a$. Moreover, if $p=2$ then necessarily $a=1$. If $p$ is odd, it is still an open problem as to whether or not $a$ must equal 1 . However, for any prime power $q$, there are known translation GQ with parameters $\left(q, q^{2}\right)$. For all these translation GQ $\mathcal{S}$ Jef showed that the translation dual $\mathcal{S}^{D}$ has a spread. This is a significant result that uses a fairly deep theorem of Segre. The resulting spread for the Roman translation GQ is given in detail in the spreads and ovals paper.

At the same time, the Roman GQ $\mathcal{S}$ (with parameters $\left(q, q^{2}\right)=\left(3^{e}, 3^{2 e}\right)$ ) have subquadrangles of order $q$ that are isomorphic to the parabolic quadric $Q\left(4,3^{e}\right)$. Each point of $\mathcal{S}$ not in a particular subquadrangle $Q\left(4,3^{e}\right)$ is collinear with the $1+3^{2 e}$ points of an ovoid of $Q\left(4,3^{e}\right)$. These ovoids were new at the time the spreads and ovoids paper appeared. Something I find quite interesting is that there are only $q^{2}\left(q^{2}-1\right)$ points of $\mathcal{S} \backslash Q(4, q)$, but the ovoids of $Q(4, q)$ determined by the points of $\mathcal{S} \backslash Q(4, q)$ all belong to a single orbit of size $q^{2}\left(q^{2}-1\right)\left(q^{4}-1\right) / 4$ 
under the action of $P \Gamma O(5, q)$. So the set of ovoids equivalent to the subtended ovoids is a set of ovoids that is $\left(q^{4}-1\right) / 4$ times as large as the set of subtended ovoids.

Now I will mention a small number of open problems that I would like to see solved.

Problem 1. If $q$ is a power of a prime, and if there is a translation $G Q\left(q^{a}, q^{a+1}\right)$ (with a necessarily odd), is it necessarily the case that $a=1$ ?

This problem has bugged me ever since I proved in [Pa83] that this is the case if $q$ is even. Several mathematicians have worked on this problem with interesting partial results, but it seems to be very stubborn.

Recall that J. Tits first constructed a $G Q(q, q)$ starting with an oval in $P G(2, q)$. That construction was extended by various authors to yield the following. Let $k \in \mathcal{Q}=\{q, q+1, q+2\}$ and let $\mathcal{K}$ be any $k$-arc in the Desarguesian plane $P G(2, q)$ embedded in $P G(3, q)$. Then there is a way to construct a generalized quadrangle whose parameters are in the set

$$
\mathcal{P}=\{(q+1, q-1),(q, q),(q-1, q+1)\} .
$$

When $q$ is even there are several nonisomorphic GQ that arise in each case just by starting with inequivalent arcs. However, when $q$ is a power of an odd prime, then (up to duality in case $k=q+1$ ) only one example is known. This suggests another open problem of interest.

Problem 2. For some odd prime power q find a non-classical $G Q(s, t)$ for some $(s, t) \in \mathcal{P}$.

The point-collinearity graph of any GQ is a strongly regular graph, so that for $k \in \mathcal{Q}$ there is a way to start with a $k$-arc in $P G(2, q)$, construct a GQ, and then construct the strongly regular graph. What seems to be new is that one can start with a $k$-arc, $k \in \mathcal{Q}$, in any finite projective plane with order $q$ and bypass the construction of a GQ to get the strongly regular graph. Then one can investigate whether or not the strongly regular graph is actually geometric, i.e., whether or not it comes from an appropriate GQ. This is the approach taken in current research jointly undertaken with Tim Penttila and his recent Ph.D. student Liz Lane-Harvard. For this moment I will give the parameters of the resulting strongly regular graphs but will give a detailed construction in only one case.

Starting with a $\mathrm{GQ}(s, t)$ for some $(s, t) \in \mathcal{P}$, one can construct a symmetric block design. This construction is already in the red book. This symmetric block design can be used to construct a strongly regular graph as was clear to Ahrens 
and Szekeres back in 1971. But if the GQ were constructed starting with a $k$-arc in $\pi_{\infty}=P G(2, q)$ for $k \in\{q, q+1, q+2\}$ it seems to have been overlooked that one could describe the strongly regular graph starting with the $k$-arc and without using the fact that classically it lived in a desarguesian plane. What the three of us have started to study is how to start with the arc in an arbitrary finite projective plane, build the strongly regular graph and then try to construct a GQ whose point-collinearity graph would be the original strongly regular graph. For $k \in\{q, q+1, q+2\}$ and any $k$-arc in any plane $\pi$ of order $q$ the strongly regular graph is straight forward to construct. On this occasion we just give the detailed construction for one example.

Theorem. Let $\mathcal{A}$ be any affine plane of order $q$ and let $\mathcal{K}$ be a $k$-arc in $\mathcal{A}, k \in \mathcal{Q}$.

1. If $k=q$, then there is a strongly regular graph

$$
\Gamma(\mathcal{K})=\operatorname{srg}\left(q^{3}, q^{2}+q-2, q-2, q+2\right)
$$

described as follows:

The vertices of $\Gamma(\mathcal{K})$ are the ordered pairs in $\mathcal{A} \times \mathcal{K}$. Adjacency is defined as follows. Distinct vertices $(P, A)$ and $(Q, B)$ are adjacent provided

(a) $P=Q$ and $A \neq B$, i.e., $(P, A) \sim(P, B)$ for distinct $A, B \in \mathcal{K}$; or

(b) $P \neq Q, A \neq B$ and $P Q$ is parallel to $A B$; or

(c) $P \neq Q, A=B$ and $P Q$ is parallel to one of the two lines tangent to $\mathcal{K}$ at $A$.

A routine case by case analysis shows that $\Gamma(\mathcal{K})$ is indeed a strongly regular graph with the parameters given in the theorem.

Our primary interest at the moment is in the case where $q$ is odd. Sadly, the more I look at the problem the less optimistic I become about constructing new GQ from these graphs, but perhaps the strongly regular graphs are of some independent interest. (See the addendum for a proof that the strongly regular graph exists for any $q$-arc in any affine plane of order $q$.)

2. Let $k=q+1$. Then there is a strongly regular graph

$$
\Gamma(\mathcal{K})=\operatorname{srg}\left(q^{3}+q^{2}+q+1, q^{2}+q, q-1, q+1\right) .
$$

3. Let $k=q+2$ (forcing $q$ to be even). Then there is a strongly regular graph

$$
\Gamma(\mathcal{K})=\operatorname{srg}\left(q^{3}+2 q^{2}, q^{2}+q, q, q\right) .
$$


Our next problem is one that was in the spotlight for a short time many years ago when our late friend Bob Liebler thought he had a chance of solving it affirmatively. But it seems to be a hard problem that will require new techniques.

Problem 3. If there is a $G Q(s, t)$, must it be true that $s \equiv t(\bmod 2)$ ?

There are perhaps silly "little" problems that nevertheless are frustrating.

Problem 4. Determine all GQ with relatively small parameters. The following cases are of special interest to me.

(i) Determine all GQ of order 5.

Bart De Bruyn has recently shown that any GQ of order 5 with at least one regular point must be the classical symplectic geometry $W(5)$ in $P G(3,5)$. It is also true by old results of Mazzoca and independently by Payne and Thas that a GQ having odd order 5 with even one antiregular point must be the classical quadric $Q(4,5)$, which is the point-line dual of $W(5)$.

(ii) Show that there is no GQ with order 6.

We know that such a GQ could not have a regular point, since there is no projective plane of order 6 . It could not have an antiregular point, since 6 is even. Also, it cannot be self-dual by a result of Willem Haemers.

(iii) Determine all $G Q(4,16)$.

A number of published results relate to this problem, but it seems still out of reach.

Problem 5. Show that no GQ of odd order can be self-dual.

This is certainly true of the known GQ of odd order, but it might be possible to prove it in general. I do not even know how to prove that a GQ of order 5 cannot be self-dual.

The next problem I mention is somewhat open ended.

Problem 6. W. Kantor has given us a way to describe what is called an elation generalized quadrangle as a kind of coset geometry. This particular approach has contributed to the construction and/or description of many families of GQ. But why should it be the only way? Already in the red book the notion of 4-gonal partition was studied briefly and more recently investigated by Dina Ghinelli [Gh12]. Her paper has a satisfying characterization of two types of GQ that would arise from a 4-gonal partition, but there is no attempt to produce new examples.

In a related study John Bamberg and Michael Giudici [BG10] have shown that the GQ with parameters $(q-1, q+1)$ obtained from the symplectic GQ denoted 
$W(q)$ by a process called Payne-derivation admit at least two non isomorphic pointregular groups, and in a few cases at least three such groups. Recent work by S. De Winter and $K$. Thas carries these ideas much further.

So far as I am aware, no successful study of $G Q\left(q^{2}, q^{3}\right)$ in terms of groups has been presented in such a way that new examples could be sought.

In two of the infinite families of known flock GQ (see [Pa98]) there is a nonabelian group $\mathcal{T}$ of collineations acting sharply transitively on the lines not concurrent with a specific line [ $\infty]$. I always hoped that someone would find a way to start with such a group and recover the entire GQ. This might lead to an interesting alternative way to describe those two families so that perhaps there would be a whole new type of construction of GQ starting with groups.

The next problem I mention might appear not to deal directly with GQ, but it really does. Recall that J. Tits has constructed a translation GQ with parameters $\left(q, q^{2}\right)$ starting with any ovoid in $P G(3, q)$.

Problem 7. Show that all ovoids in $P G(3, q)$ are known.

Or give us all a very pleasant surprise by finding a new one!

Problem 8. Let $\mathcal{S}$ be a $G Q$ of odd order $q$ with an antiregular point $p$ (i.e., every triad of points containing $p$ has exactly zero or two centers). Bagchi, Brouwer and Wilbrink have shown us that then every point of $\mathcal{S}$ is antiregular. This implies that many projective planes can be constructed. I believe that it should be possible to show that $\mathcal{S}$ must be isomorphic to the classical $Q(4, q)$.

\section{Addendum: A Family of Strongly Regular Graphs}

I remind the reader that this addendum is joint work with Tim Penttila and Liz Lane-Harvard.

Let $\mathcal{A}$ be a finite affine plane of order $q$ with point set $\mathcal{P}$ containing a $q$-arc $K$. Define a graph $\Gamma(\mathcal{A}, K)$ as follows. The vertex set $\mathcal{V}$ is $\mathcal{P} \times K$ and adjacency is defined by $(P, A) \sim(Q, B)$ if and only if

(i) $A \neq B$ and either $P=Q$ or $P Q \| A B$, or

(ii) $A=B, P \neq Q$, and $P Q$ is parallel to one of the two tangent lines to $K$ at the point $A$.

Theorem. The graph $\Gamma(\mathcal{A}, K)$ defined above is a strongly regular graph with parameters $\left(q^{3}, q^{2}+q-2, q-2, q+2\right)$. 
Proof. Throughout this proof the letters $P, Q, X, Y$ will denote general points of $\mathcal{P}$ and the letters $A, B, C$ will denote points of $K$.

The number of vertices is clearly $q^{3}$. Let $(P, A) \in \mathcal{V}$. There are $q-1$ points $B$ of $K \backslash\{A\}$, so $q-1$ vertices $(P, B) \sim(P, A)$. If $B \neq A$, let $\ell$ be the line of $\mathcal{A}$ through $P$ parallel to $A B$. There are $q-1$ points of $\ell \backslash\{P\}$, so $q-1$ vertices $(Q, B) \sim(P, A)$. So incidence condition (i) accounts for $q-1+(q-1)(q-$ $1)=q(q-1)$ vertices $(Q, B)$ with $(P, A) \sim(Q, B)$. To see the contribution of condition (ii), note that there are two lines of $\mathcal{A}$ through $A$ tangent to $K$, and $q-1$ points $Q$ different from $P$ on each of the two lines through $P$ parallel to a tangent through $A$, giving $2(q-1)$ vertices $(Q, A) \sim(P, A)$. Hence the valency of the graph $\Gamma(\mathcal{A}, K)$ is $q(q-1)+2(q-1)=q^{2}+q-2$.

We now consider three cases where two vertices are adjacent and we count their common neighbors.

Case 1. If $A \neq B$, so $(P, A) \sim(P, B)$, there are $q-2$ common neighbors of the form $(P, C)$ with $C \in K \backslash\{A, B\}$. Consider whether or not there could be a common neighbor of the form $(X, C)$ with $X \neq P$.

Let $\ell_{A}$ be the line through $A$ parallel to $P X, \ell_{B}$ the line through $B$ parallel to $P X$. Then

- $(X, C) \sim(P, A)$ if and only if $A \neq C$ and $\ell_{A}=A C$, or $A=C$ and $P X$ is parallel to a tangent at $A$.

- $(X, C) \sim(P, B)$ if and only if $B \neq C$ and $\ell_{B}=B C$ or $B=C$ and $P X$ is parallel to a tangent at $B$.

First note that we cannot have $A \neq C \neq B$, for then $\ell_{A}=A C=B C$, which contradicts the fact that $K$ is an arc. So either $A=C$ or $B=C$. Without loss of generality, suppose $A=C$. Then $\ell_{B}=B C$ with $P X$ parallel to a tangent at $A$ and also parallel to $A B$ which is not a tangent at $A$. Hence if $A \neq B$, then $(P, A)$ and $(P, B)$ have exactly $q-2$ common neighbors.

Case 2. Suppose that $A \neq B, P \neq Q$ and $P Q \| A B$, so $(P, A) \sim(Q, B)$. Let $X, C)$ be a common neighbor. First suppose that $X=P$. So $(P, C) \sim(P, A)$ implies $C \neq A$. Then $(P, C) \sim(Q, B)$ with $P \neq Q$ implies that $P Q \| B C$. This forces $B A=B C$, which implies $A=C$ since $K$ is an arc. This contradiction shows that $X \neq P$. Similarly, $X \neq Q$.

So now we have $P \neq X \neq Q$ and still have $P Q \| A B$. Let $\ell_{A}$ be the line through $A$ parallel to $P X ; \ell_{B}$ be the line through $B$ parallel to $Q X$.

- $(X, C) \sim(P, A)$ if and only if $A \neq C$ and $\ell_{A}=A C$, or $A=C$ and $P X$ is parallel to a tangent at $A$. 
- $(X, C) \sim(Q, B)$ if and only if $B \neq C$ and $\ell_{B}=B C$, or $B=C$ and $Q X$ is parallel to a tangent at $B$.

Since we cannot have both $C=A$ and $C=B$, assume without loss of generality that $C \neq A$ and $A C$ is the line through $A$ parallel to $P X$. First suppose that $C=B$ and $Q X$ is parallel to a tangent at $B$. Then $\ell_{A}=A C=A B$ is the line through $A$ parallel to $P X$. But $A B$ is now parallel to both $P Q$ and $P X$, implying that $X$ is on $P Q$. Then $Q X=P Q$ is parallel to $A B$, which is not a tangent through $B$. This contradiction shows that $A, B, C$ must be distinct. And we have that $A C$ is the line through $A$ parallel to $P X ; B C$ is the line through $B$ parallel to $Q X$, and $A B \| P Q$. We can now count the common neighbors. Let $C \in K \backslash\{A, B\}$. Let $\ell$ be the line through $P$ parallel to $A C$ and let $m$ be the line through $Q$ parallel to $B C$. Since $A C$ and $B C$ cannot be parallel because $K$ is an arc, clearly $\ell$ and $m$ are not parallel and meet at a unique point $X$. In this way we get exactly $q-2$ vertices $(X, C)$ which are common neighbors of $(P, A)$ and $(Q, B)$.

The last case for adjacent vertices is the following.

Case 3. $P \neq Q$ and $P Q$ is parallel to some line tangent to $K$ at a point $A$, so $(P, A) \sim(Q, A)$.

Suppose that $(X, C)$ is a common neighbor. If $X=P$, then $(P, C) \sim(P, A)$ implies $C \neq A$. Also $(P, C) \sim(Q, A)$ implies that $P Q \| C A$, which is impossible since $P Q$ is parallel to some line tangent to $K$ at $A$. It follows that $X \neq P$. Similarly $X \neq Q$.

Finally, suppose that $C \neq A$, and of course that $P \neq X \neq Q$. Then $(X, C) \sim$ $(P, A)$ implies $P X \| A C$, and $Q X \| A C$ implies $X \in P Q$, which forces $P Q \|$ $A C$. but this forces $C=A$, since $P Q$ is parallel to a tangent at $A$. It follows that the common neighbors are the vertices $(X, A)$, such that $X \in P Q \backslash\{P, Q\}$. This completes a proof that any two adjacent vertices have exactly $q-2$ common neighbors.

Case 4. $A \neq B$ and $(P, A) \nsim(Q, B)$, so $P \neq Q$ and $P Q \nVdash A B$. For each $C \in K \backslash\{A, B\}$ let $\ell$ be the line on $P$ parallel to $A C$ and $m$ the line on $Q$ parallel to $B C$. Since $A C \nVdash B C, \ell$ must meet $m$ in a point $X$. Then $(X, C)$ is a common neighbor of $(P, A)$ and $(Q, B)$. Note that $P Q \| B C$ if and only if $(X, C)=(P, C)$. Similarly, $P Q \| A C$ if and only if $(X, C)=(Q, C)$. So far this yields $q-2$ common neighbors of $(P, A)$ and $(Q, B)$.

Lemma. For each $C \in K \backslash\{A, B\}$ there is a unique vertex $(X, C)$ that is a common neighbor of $(P, A)$ and $(Q, B)$. 
Proof. So far we have one $X$ for each such $C$. Now suppose we have the following. $A, B, C$ are distinct points of $K ; P \neq Q ; P Q \nVdash A B ; X \neq Y ;(X, C)$ and $(Y, C)$ are common neighbors of $(P, A)$ and $(Q, B)$. This last assumption is equivalent to the following four conditions holding:
(a) $P=X$ or $P X \| A C$,
(b) $Q=X$ or $Q X \| B C$,
(c) $P=Y$ or $P Y \| A C$,
(d) $Q=Y$ or $Q Y \| B C$.

These assumptions are symmetric in $P$ and $Q$ and also in $X$ and $Y$. We first assume that one of $P, Q$ equals one of $X, Y$. Without loss of generality we assume that $P=X$, so $P Q \| B C$. Also $Q \neq X$ implies $Q X \| B C$. If $Q$ were equal to $Y$, then $P Q \| A C$. But $P Q \| B C \nVdash A C$. So $Q \neq Y$ implies $Q Y \| B C$. So we have $P Q\|B C\| Q Y$, which implies $Y$ is on $P Q$. But then we have $A C\|P Y\| B C$, which is impossible since $K$ is an arc.

This means we must have that $P, Q, X, Y$ are all distinct. So we have $P X \|$ $A C, Q X\|B C, P Y\| A C$, and $Q Y \| B C$. Then $P X \| P Y$ and $Q X \| Q Y$ says that $P, X, Y$ are collinear and $Q, X, Y$ are collinear. This leads to $A C\|P X\|$ $Q Y \| B C$, a contradiction.

Finally, we consider what common neighbors of the form $(X, A)$ or $(X, B)$ could arise. $(X, A) \sim(P, A)$ implies $P X$ is parallel to a tangent to $K$ at $A$. Since $P Q \nVdash A B$, the line $\ell$ on $Q$ parallel to $A B$ does not contain $P$. If $m$ is either one of the two lines on $P$ parallel to a tangent to $K$ at $A$, then $X=\ell \cap m$ gives a vertex $(X, A)$ that is a common neighbor of $(P, A)$ and $(Q, B)$. Similarly, there are two common neighbors of the form $(X, B)$. This completes a proof of Case 4.

Case 5. $A=B$ with $(P, A) \nsim(Q, A), P \neq Q$. We first consider whether there could be a common neighbor of the form $(X, A)$. In this case we have to have $P \neq X \neq Q$ and $P X$ parallel to a tangent at $A, Q X$ parallel to a tangent at $A$. If $\ell$ is one tangent at $A$ and $m$ is the other, let $\ell_{P}$ be the line on $P$ parallel to $\ell$ and $m_{P}$ the line on $P$ parallel to $m$. Similarly, $\ell_{Q}$ and $m_{Q}$ are the lines on $Q$ parallel to $\ell$ and $m$, respectively. Since $P Q$ is not parallel to either $\ell$ or $m$ by hypothesis, we see that $\ell_{P}$ must meet $m_{Q}$ at a point $X_{1}$ and $m_{P}$ must meet $\ell_{Q}$ at a point $X_{2}$, giving two common neighbors $\left(X_{1}, A\right)$ and $\left(X_{2}, A\right)$.

Finally, we consider common neighbors of the form $(X, C)$ where $C \neq A$. Here we have $P=X$ or $P X \| A C$ and $Q=X$ or $Q X \| A C$. In either case we end up with $P Q \| A C$. Since we are given that $P Q$ is not parallel to one of the tangents at $A$, it must be that $P Q$ is parallel to a unique secant $A C, C \in K$. So if $P \neq X \neq Q, P X\|A C\| P Q$ implies $X$ lies on the line $P Q$. Conversely, for 
any point $X$ on $P Q$, we see that $(X, C)$ is a common neighbor. In all, this give $2+q$ common neighbors of $(P, A)$ and $(Q, A)$.

This completes a proof that two vertices that are not adjacent have exactly $q+2$ common neighbors. Hence the theorem is proved.

\section{References}

[BG10] J. Bamberg and M. Giudici, Point-regular automorphism groups of generalized quadrangles, J. Combin. Theory Ser. A 118 (2011), 11141128.

[Gh12] D. Ghinelli, Characterization of some 4-tonal configurations of Ahrens-Szekeres type, European J. Combin. 33 (2012), 1557-1573.

[Pa83] S. E. Payne, On the Structure of Translation Generalized Quadrangles, Ann. Discrete Math. 18 (1983), 661-666.

[Pa98] _ A problem on generalized quadrangles, J. Statist. Plann. Inference 73 (1998), 197-204.

[PT84] S. E. Payne and J. A. Thas, Finite Generalized Quadrangles, Pitman Research Notes in Mathematics 110, 1984.

[PT09] _ Finite Generalized Quadrangles, Second edition, EMS Series of Lectures in Mathematics 9, European Mathematical Society (EMS), Zürich, 2009.

[PT94] Dedicata 52 (1994), 227-253. 